\title{
Heuristic rating estimation: geometric approach
}

\author{
Konrad Kułakowski · Katarzyna Grobler-Dębska • \\ Jarosław Wąs
}

Received: 30 May 2014 / Accepted: 28 October 2014 / Published online: 9 November 2014

(C) The Author(s) 2014. This article is published with open access at Springerlink.com

\begin{abstract}
Heuristic rating estimation is a newly proposed method that supports decisions analysis based on the use of pairwise comparisons. It allows the ranking values of some alternatives (herein referred to as concepts) to be initially known, whilst ranks for other concepts have yet to be estimated. To calculate the missing ranks it is assumed that the priority of every single concept can be determined as the weighted arithmetic mean of the priorities of all the other concepts. It has been shown that the problem has an admissible solution if the inconsistency of the pairwise comparisons is not too high. The proposed approach adopts heuristics according to which a weighted geometric mean is used to determine the missing priorities. In this approach, despite increased complexity, a solution always exists and its existence does not depend on the inconsistency or reciprocity of the input matrix. Thus, the presented approach might be appropriate for a larger number of problems than previous methods. Moreover, it turns out that the geometric approach, as proposed in the article, can be optimal. The optimality condition is presented in the form of a corresponding theorem. A formal definition of the proposed geometric heuristics is accompanied by two numerical examples.
\end{abstract}

Keywords Multicriteria analysis · Pairwise comparisons · Ranking theory · AHP . Logarithmic least square method

This research is supported by AGH University of Science and Technology, Contract No.: 11.11.120.859.

K. Kułakowski $(\bowtie) \cdot$ K. Grobler-Dębska $\cdot$ J. Wąs

AGH University of Science and Technology, al. Mickiewicza 30, Kraków, Poland

e-mail: konrad.kulakowski@agh.edu.pl

K. Grobler-Dębska

e-mail: grobler@agh.edu.pl

J. Wąs

e-mail: jaroslaw.was@agh.edu.pl 


\section{Introduction}

The first written evidence for the pairwise comparisons (PC) method dates back to the thirteenth century, when Ramon Llull from Majorca wrote a seminal piece "Artifitium electionis personarum" (The method for the elections of persons) about voting and elections [5,7], followed by two subsequent works forming a practical study on election processes. ${ }^{1}$ Nowadays, $\mathrm{PC}$ as a voting method is a way of deciding on the relative utility of alternatives used in decision theory [25] and other fields like economics [22], psychometrics and psychophysics [28] and so on. PC theory is developed by many research teams in different fields and approaches. Characteristic approaches can be noted, such as AHP-analytic hierarchy process proposed and constantly developed by Saaty [25,27], the approximate PC reasoning [12,20], the issue of incomplete PC relations [1], nonreciprocal PC relation properties [8], and the problem of non-numerical rankings [11]. There are also many case studies and applications $[13,19]$.

The heuristic rating estimation (HRE) method which enables the user to explicitly define a reference set of concepts, for which the ranking values are a priori known, is currently being developed [16,17]. The base heuristics used in HRE proposes to determine the relative values of a single non-reference concept as a weighted arithmetic mean of all the other concepts. This proposition leads to the linear equation system defined by the matrix $\mathbf{A}$ and the strictly positive vector of constant terms $b$.

In this work, the authors show that using a geometric mean, rather than an arithmetic one, to determine the relative priorities of concepts may in some cases be more convenient. The main benefit of the proposed solution stems from the guarantee of the existence of a solution. ${ }^{2}$ Hence, unlike the original proposal, a ranking list can be created for all even a nonreciprocal matrices. This guarantee is paid for by an increase in computational complexity. The presented solution is accompanied by two numerical examples.

The HRE approach can potentially be useful in many situation when the reference data are available. In particular it might be interesting for the AHP users as a viable alternative for the eigenvalue based [25] or the geometric mean method [6].

The presented work is a continuation of research initiated in [16,17]. It redefines the main heuristics of HRE and the method of calculating the solution. The HRE approach proposed in the previous articles is briefly outlined in (Sect. 2). There is also a short summary of a number of important properties of M-matrices (Sect. 2.3), which are essential to the properties of the presented method. The next section (Sect. 3) describes the proposed solution and discusses two important properties: solution existence (Sect. 3.2) and optimality (Sect. 3.3). These theoretical considerations are accompanied by two meaningful examples showing how the presented method can be used in practice (Sect. 4). A brief summary is provided in (Sect. 5).

\section{Preliminaries}

\subsection{Basic concepts of pairwise comparisons method}

The input for the $P C$ method is the $P C$ matrix $M=\left(m_{i j}\right)$, where $m_{i j} \in \mathbb{R}_{+}$and $i, j \in$ $\{1, \ldots, n\}$. The values $m_{i j}$ and $m_{j i}$ represent subjective expert judgments as to the relative

\footnotetext{
1 See: The Augsburg Web Edition of Llull's Electoral Writings.

2 It has been shown that for a reasonably small inconsistency the original (additive) HRE approach [17] also provides a feasible solution [18].
} 
importance, utility or quality indicators of concepts $c_{i}$ and $c_{j}$. Thus, according to the best knowledge of experts, it should hold $c_{i}=m_{i j} c_{j}$.

Definition 1 A matrix $M$ is said to be reciprocal if for all $i, j \in\{1, \ldots, n\}$ it holds that $m_{i j}=\frac{1}{m_{j i}}$, and $M$ is said to be consistent if for all $i, j, k \in\{1, \ldots, n\}$ is $m_{i j} \cdot m_{j k} \cdot m_{k i}=1$.

Since the data in the $P C$ matrix represents subjective opinions of experts, they might be inconsistent. Hence, there may exist a triad $m_{i j}, m_{j k}, m_{k i}$ of entries in $M$ for which $m_{i k} \cdot m_{k j} \neq$ $m_{i j}$. This leads to a situation in which the relative importance of $c_{i}$ with respect to $c_{j}$ is either $m_{i k} \cdot m_{k j}$ or $m_{i j}$. This observation underlies two related concepts: a priority deriving method that transforms even an inconsistent matrix $M$ into a consistent priority vector, and an inconsistency index that describes to what extent the matrix $M$ is inconsistent. The problem of inconsistency has been addressed by Saaty in his seminal work [25], later intensively studied also by other researchers $[4,14]$. There are a number of priority deriving methods [9] and inconsistency indexes [2,3]. Following Saaty a good inconsistency indicator is the principal eigenvalue of $M$.

Definition 2 Saaty's inconsistency index $\mathscr{S}$ of $n \times n$ and $(n>2)$ reciprocal matrix $M$ is equal to

$$
\mathscr{S}(M) \stackrel{d f}{=} \frac{\lambda_{\max }-n}{n-1}
$$

where $\lambda_{\max }$ is the principal eigenvalue of $M$.

According to [25] the matrix is sufficiently consistent when $\mathscr{S}(M) \leq 0.1$ (the usual limit of acceptability for the matrix $4 \times 4$ is 0.08 [see [26]]).

The result of the pairwise comparisons method is a ranking-a function that assigns values to the concepts. Formally, it can be defined as follows.

Definition 3 The ranking function for $C$ (the ranking of $C$ ) is a function $\mu: C \rightarrow \mathbb{R}_{+}$that assigns to every concept from $C$ a positive value from $\mathbb{R}_{+}$.

Thus, $\mu(c)$ represents the ranking value for $c \in C$. The $\mu$ function is usually defined as a vector of weights $\mu \stackrel{d f}{=}\left[\mu\left(c_{1}\right), \ldots, \mu\left(c_{n}\right)\right]^{T}$. According to the most popular eigenvalue based approach proposed by Saaty [25] the final ranking $\mu_{e v}$ is determined as the principal eigenvector of the $P C$ matrix $M$, rescaled so that the sum of all its entries is 1, i.e.

$$
\mu_{e v}=\left[\frac{\mu_{\max }\left(c_{1}\right)}{s_{e v}}, \ldots, \frac{\mu_{\max }\left(c_{n}\right)}{s_{e v}}\right]^{T} \text { and } s_{e v}=\sum_{i=1}^{n} \mu_{\max }\left(c_{i}\right)
$$

where $\mu_{e v}$-the ranking function, $\mu_{\max } \stackrel{d f}{=}\left[\mu_{\max }\left(c_{1}\right), \ldots, \mu_{\max }\left(c_{n}\right)\right]^{T}$ - the principal eigenvector of $M$. Another popular approach proposes the rescaled geometric mean (GM) of rows of $M$ as the ranking result, i.e.

$$
\mu_{g m}=\left[\frac{p_{1}}{s_{g m}}, \ldots, \frac{p_{n}}{s_{g m}}\right]^{T}
$$

where

$$
p_{i}=\left(\prod_{j=1}^{n} m_{i j}\right)^{\frac{1}{n}} \text { and } s_{g m}=\sum_{i=1}^{n}\left(\prod_{j=1}^{n} m_{i j}\right)^{\frac{1}{n}}
$$

It can be shown that for the fully consistent matrix $M$ the ranking vectors $\mu_{e v}$ and $\mu_{g m}$ are identical. 
The geometric mean minimizes the sum of logarithmic errors $e[6,10]$ given as:

$$
e\left(\mu\left(c_{1}\right), \ldots, \mu\left(c_{n}\right)\right)=\sum_{i=1}^{n} \sum_{j=1}^{n}\left(\ln \left(m_{i j}\right)-\ln \left(\frac{\mu\left(c_{i}\right)}{\mu\left(c_{j}\right)}\right)\right)^{2}
$$

Hence, a direct solving of a logarithmic least squares optimization problem can also be used as the priority deriving procedure. As it will be shown later in (Sect. 3.3) under certain conditions also the presented geometric approach minimizes (5). Thus, despite the fact that the presented approach is a ranking procedure, it can serve as a method of solving constrained optimization problem.

A more complete overview including other priority deriving methods can be found in $[2,9]$.

\subsection{Pairwise comparisons method with the reference set}

Usually, when using the pairwise comparisons method, the ranking values $\mu\left(c_{1}\right), \ldots, \mu\left(c_{n}\right)$ are initially unknown. Hence, they need to be determined by the priority deriving procedure. In some cases, however, there are concepts for which the priorities are known from elsewhere. Hence, the decision makers may have additional knowledge about the group of elements $C_{K} \subseteq C$ that allows them to determine $\mu(c)$ for $C_{K}$ in advance.

For example, let $c_{1}, c_{2}$ and $c_{3}$ represent oil paintings that an auction house plans to put up for auction. The sequence of paintings during the auction should correspond to their approximate valuation. In order to determine the indicative price of paintings the auction house asked experts to evaluate them in pairs, taking into account that two other paintings from the same period of time were previously auctioned for $\mu\left(c_{4}\right)$ and $\mu\left(c_{5}\right)$.

The situation as described above prompted the first author [16,17] to propose a heuristic rating estimation (HRE) model. According to HRE the set of concepts $C$ is composed of unknown concepts $C_{U}=\left\{c_{1}, \ldots, c_{k}\right\}$ and known (reference) concepts $C_{K}=\left\{c_{k+1}, \ldots, c_{n}\right\}$, where $C_{U}, C_{K} \neq \emptyset$ and $C_{U} \cap C_{K}=\emptyset$. The values $\mu\left(c_{i}\right)$ for $c_{i} \in C_{K}$ are known, whilst the values $\mu\left(c_{j}\right)$ for elements $c_{j} \in C_{U}$ need to be calculated. Following the heuristics of averaging with respect to the reference values [17], the solution proposed by HRE is to adopt as $\mu\left(c_{j}\right)$, for every $c_{j} \in C_{U}$, the arithmetic mean of all the other values $\mu\left(c_{i}\right)$ multiplied by factor $m_{j i}$ :

$$
\mu\left(c_{j}\right)=\frac{1}{n-1} \sum_{i=1, i \neq j}^{n} m_{j i} \mu\left(c_{i}\right)
$$

If the expert judgments gathered in the matrix $M$ were fully consistent (Definition 1), then every component of the sum (6) in the form $m_{j i} \mu\left(c_{i}\right)$ would equal $\mu\left(c_{j}\right)$. Because they are generally not, then every component is only an approximation of $\mu\left(c_{j}\right)$. Thus, the arithmetic mean of the individual approximations has been adopted as the most probable value of $\mu\left(c_{j}\right)$. To determine unknown values $\mu\left(c_{j}\right)$ for $c_{j} \in C_{U}$ the problem, formalised as (6), can be written down as the linear equation system $A \mu=b$, where:

$$
A=\left[\begin{array}{ccc}
1 & \cdots & -\frac{1}{n-1} m_{1, k} \\
-\frac{1}{n-1} m_{2,1} & \cdots & -\frac{1}{n-1} m_{2, k} \\
\vdots & \ddots & \vdots \\
-\frac{1}{n-1} m_{k, 1} & \cdots & 1
\end{array}\right]
$$


and

$$
b=\left[\begin{array}{c}
\frac{1}{n-1} \sum_{i=k+1}^{n} m_{1, i} \mu\left(c_{i}\right) \\
\frac{1}{n-1} \sum_{i=k+1}^{n} m_{2, i} \mu\left(c_{i}\right) \\
\vdots \\
\frac{1}{n-1} \sum_{i=k+1}^{n} m_{k, i} \mu\left(c_{i}\right)
\end{array}\right]
$$

The solution $\mu=\left[\mu\left(c_{1}\right), \ldots, \mu\left(c_{k}\right)\right]^{T}$ determines the values of $\mu$ for elements from $C_{U}$. Together with the known $\mu\left(c_{k+1}\right), \ldots, \mu\left(c_{n}\right)$, the vector $\mu$ forms the complete result list, which after sorting can be used to build a ranking. Although the values $\mu(c)$ for $c \in C$ are called priorities, they usually have a specific meaning. In the case of the previously mentioned example they represent the expected price of paintings.

According (Definition 3) the ranking results must be strictly positive, hence only strictly positive vectors $\mu$ are considered to be feasible. It can be shown that the equation $A \mu=b$ has a feasible solution if $A$ is strictly diagonally dominant by rows [17]. It has recently been shown [18] that the equation has a feasible solution when the Koczkodaj's inconsistency index [14] is not to high.

\subsection{M-matrices}

Very often the real life problem can be reduced to the linear equation system $A \mu=b$, where the matrix $A$ has some special structure. Frequently the matrix $A$ has positive diagonal and nonpositive off-diagonal entries. Due to their importance in practice this type of matrix has been especially thoroughly studied by researchers $[23,24]$. To define it formally a few more notions and definitions are needed.

Let $\mathscr{M}_{\mathbb{R}}(n)$ be a set of $n \times n$ matrices over $\mathbb{R}$, and $\mathscr{M}_{\mathbb{Z}}(n)$ the set of all $A=\left[a_{i j}\right] \in \mathscr{M}_{\mathbb{R}}(n)$ with $a_{i j} \leq 0$ if $i \neq j$ and $i, j \in\{1, \ldots, n\}$. Furthermore, assume that for every matrix $A \in \mathscr{M}_{\mathbb{R}}(n)$ and vector $b \in \mathbb{R}^{n}$ the notation $A \geq 0$ and $b \geq 0$ will mean that every $m_{i j}$ and $b_{k}$ are non-negative and neither $A$ nor $b$ equals 0 . The spectral radius of $A$ is defined as $\rho(A) \stackrel{d f}{=} \max \{|\lambda|: \operatorname{det}(\lambda I-A)=0\}$.

Definition 4 An $n \times n$ matrix that can be expressed in the form $A=s I-B$ where $B=\left[b_{i j}\right]$ with $b_{i j} \geq 0$ for $i, j \in\{1, \ldots, n\}$, and $s \geq \rho(B)$, the maximum of the moduli of the eigenvalues of $\mathrm{B}$, is called an M-matrix.

Following [23] some M-matrix properties are recalled below in the form of the Theorem 1.

Theorem 1 For every $A \in \mathscr{M}_{\mathbb{Z}}(n)$ each of the following conditions is equivalent to the statement: $A$ is a nonsingular M-matrix.

1. $A$ is inverse positive. That is, $A^{-1}$ exists and $A^{-1} \geq 0$

2. There exists a positive diagonal matrix $D$ such that $A D$ has all positive row sums.

It is worth noting that for every matrix equation in the form $A \mu=b$, where $A$ is a nonsingular M-matrix, $\mu=A^{-1} b$ holds. Since $A^{-1} \geq 0, b>0$ also implies that $\mu>0$.

\section{HRE: geometric approach}

3.1 Heuristics of the geometric averaging with respect to the reference values

The pairwise comparisons method is most often used to transform a $P C$ matrix into a ranking list of mutually compared concepts. During the transformation a priority is assigned to each 
concept. Therefore, this transformation is often called a priority deriving method. There are many priority deriving methods. Besides the eigenvalue based method (2), where the ranking values $\mu\left(c_{i}\right)$ are approximated as the arithmetic means of $m_{i j} \cdot \mu\left(c_{j}\right)$, the geometric mean of rows is also used (3). This may suggest that for a ranking problem with the reference set [17], the arithmetic mean (6) might also be replaced by the geometric mean. This observation prompted the author to formulate and investigate geometric averaging with respect to reference values heuristics. According to this proposition the following non-linear equation is used to determine the unknown values $\mu\left(c_{j}\right)$ for $c_{j} \in C_{U}$ :

$$
\mu\left(c_{j}\right)=\left(\prod_{i=1, i \neq j}^{n} m_{j i} \mu\left(c_{i}\right)\right)^{\frac{1}{n-1}}
$$

After raising both sides to the power of $n-1$ the geometric averaging heuristics equation (9) leads to a non-linear equation system in the form:

$$
\begin{aligned}
& \mu^{n-1}\left(c_{1}\right)=m_{1,2} \mu\left(c_{2}\right) \cdot \ldots \ldots \ldots \ldots \ldots \ldots \cdot m_{1, n} \mu\left(c_{n}\right) \\
& \mu^{n-1}\left(c_{2}\right)=m_{2,1} \mu\left(c_{1}\right) \cdot m_{2,3} \mu\left(c_{3}\right) \cdot \ldots \cdot m_{2, n} \mu\left(c_{n}\right) \\
& \mu^{n-1}\left(c_{k}\right)=m_{k, 1} \mu\left(c_{1}\right) \cdot \ldots \ldots \ldots \ldots \cdot m_{k, n-1} \mu\left(c_{n-1}\right)
\end{aligned}
$$

Of course, since the ranking values for $c_{k+1}, \ldots, c_{n} \in C_{K}$ make a reference set where the values $\mu\left(c_{j}\right)$ are known and fixed, some products in the form $m_{j i} \mu\left(c_{i}\right)$ are initially known constants. Let us denote:

$$
g_{j}=\prod_{i=k+1}^{n} m_{j i} \mu\left(c_{i}\right)
$$

for $j=1, \ldots, k$ as the constant part of each Eq. (10). Thus, the non-linear equation system can be written as:

$$
\begin{aligned}
& \mu^{n-1}\left(c_{1}\right)=m_{1,2} \mu\left(c_{2}\right) \cdot \ldots \ldots \ldots \ldots \ldots \ldots \cdot m_{1, k} \mu\left(c_{k}\right) \cdot g_{1} \\
& \mu^{n-1}\left(c_{2}\right)=m_{2,1} \mu\left(c_{1}\right) \cdot m_{2,3} \mu\left(c_{3}\right) \cdot \ldots \cdot m_{2, k} \mu\left(c_{k}\right) \cdot g_{2} \\
& \begin{array}{l}
\ldots \ldots \ldots \ldots \ldots \ldots \ldots \ldots \ldots \ldots \ldots \ldots \ldots \ldots \ldots \ldots \\
\mu^{n-1}\left(c_{k}\right)=m_{k, k} \mu\left(c_{1}\right) \cdot \ldots \ldots \ldots \ldots \ldots \\
\left.m_{k-1}\right) \cdot g_{k}
\end{array}
\end{aligned}
$$

Hence $\mu\left(c_{j}\right), m_{i j}, g_{j} \in \mathbb{R}_{+}$, let us denote $\log _{\xi} \mu\left(c_{j}\right) \stackrel{d f}{=} \widehat{\mu}\left(c_{j}\right), \widehat{m}_{i j} \stackrel{d f}{=} \log _{\xi} m_{i j}$ and $\widehat{g}_{j} \stackrel{d f}{=} \log _{\xi} g_{j}$ for some $\xi \in \mathbb{R}_{+}$. It is easy to see that the above non-linear equation system is equivalent to the following one:

$$
\begin{aligned}
& (n-1) \widehat{\mu}\left(c_{1}\right)=\widehat{m}_{1,2}+\widehat{\mu}\left(c_{2}\right)+\cdots \cdots+\widehat{m}_{1, k}+\widehat{\mu}\left(c_{k}\right)+\widehat{g}_{1} \\
& (n-1) \widehat{\mu}\left(c_{2}\right)=\widehat{m}_{2,1}+\widehat{\mu}\left(c_{1}\right)+\cdots \cdot \cdot+\widehat{m}_{2, k}+\widehat{\mu}\left(c_{k}\right)+\widehat{g}_{2}
\end{aligned}
$$

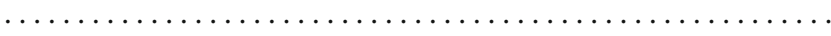

$$
\begin{aligned}
& (n-1) \widehat{\mu}\left(c_{k}\right)=\widehat{m}_{k, 1}+\widehat{\mu}\left(c_{1}\right)+\cdots+\widehat{m}_{k, k-1}+\widehat{\mu}\left(c_{k-1}\right)+\widehat{g}_{k}
\end{aligned}
$$

By grouping all the constant terms on the right side of each above equation we obtain the linear equation system

$$
\begin{aligned}
& (n-1) \widehat{\mu}\left(c_{1}\right)-\sum_{i=2}^{k} \widehat{\mu}\left(c_{i}\right)=b_{1} \\
& (n-1) \widehat{\mu}\left(c_{2}\right)-\sum_{i=1, i \neq 2}^{k} \widehat{\mu}\left(c_{i}\right)=b_{2} \\
& \ldots \ldots \ldots \ldots \ldots \ldots \ldots \ldots \ldots \ldots \ldots \ldots \ldots \ldots \ldots \ldots \ldots \ldots \\
& (n-1) \widehat{\mu}\left(c_{k}\right)-\sum_{i=1}^{k-1} \widehat{\mu}\left(c_{i}\right)=b_{k}
\end{aligned}
$$


where $b_{i} \stackrel{d f}{=} \sum_{j=1, j \neq i}^{k} \widehat{m}_{1, j}+\widehat{g}_{i}$ for $i=1, \ldots, k$, which can be easily written down in the matrix form

$$
\widehat{A} \widehat{\mu}=b
$$

where

$$
\begin{aligned}
& \widehat{A}=\left[\begin{array}{cccc}
(n-1) & -1 & \cdots & -1 \\
\vdots & \ddots & & \vdots \\
\vdots & \ddots & \vdots \\
-1 & -1 & \cdots & (n-1)
\end{array}\right], \\
& \widehat{\mu}=\left[\begin{array}{c}
\widehat{\mu}\left(c_{1}\right) \\
\widehat{\mu}\left(c_{2}\right) \\
\vdots \\
\widehat{\mu}\left(c_{k}\right)
\end{array}\right], \quad \text { and } b=\left[\begin{array}{c}
b_{1} \\
b_{2} \\
\vdots \\
b_{k}
\end{array}\right]
\end{aligned}
$$

Therefore, the solution $\widehat{\mu}$ of the linear equation system (14) automatically provides the solution to the original non-linear problem as formulated in (10). Indeed the ranking vector $\mu$ can be computed following the formula:

$$
\mu=\left[\xi^{\widehat{\mu}\left(c_{1}\right)}, \ldots, \xi^{\widehat{\mu}\left(c_{k}\right)}\right]^{T}
$$

Importantly, as is shown below, a feasible solution of (14) always exists. Hence, the heuristics of averaging with respect to the geometric mean always provides the user with an appropriate ranking function.

\subsection{Existence of solution}

The form of $\widehat{A}$ is specific. The positive diagonal and the negative off-diagonal real entries mean that $\widehat{A} \in \mathscr{M}_{\mathbb{Z}}(k)$ (see Sect. 2.3). Let us put:

$$
D=\left[\begin{array}{ccc}
1 & \cdots & 0 \\
\vdots & \ddots & \vdots \\
0 & \cdots & 1
\end{array}\right]
$$

and $D \in \mathscr{M}_{\mathbb{R}}(k)$. Of course $D$ is a positively dominant matrix. Thus, the product $\widehat{A} \cdot D=\widehat{A}$. The sum of each row in $\widehat{A}$ equals

$$
(n-1)+\sum_{i=1}^{k-1}(-1)=n-k
$$

Since $C_{K}$ is nonempty, its cardinality $\left|C_{K}\right|=n-k$ is greater than 0 . This means that the sum of each row of $\widehat{A} \cdot D$ is positive. Hence, due to the Theorem $1, \widehat{A}$ is a nonsingular M-matrix (Definition 4), the rank of $\widehat{A}$ is $k$ and also the rank of the extended matrix $[\widehat{A} \mid b]$ is $k$. Thus, $\widehat{A}^{-1}$ exists (i.e. $\widehat{\mu}=\widehat{A}^{-1} b$ ) and the equation (14) always has exactly one solution in $\mathbb{R}^{k}$. Due to the form of the solution to the main problem (17) and the fact that for any fixed $x>0$ and $y>0$ the exponential function $f(x)=x^{y}$ is a bijection in $\mathbb{R}_{+}$, the existence and uniqueness of solution of (14) implies the existence and uniqueness of solution of (10). Note that $\mu$ is a vector in $\mathbb{R}_{+}^{k}$, i.e. each of its entries is strictly positive. 
It is worth noting that, similarly as the geometric mean method (3), the presented approach provides a feasible solution also for non-reciprocal matrices. In other words, unlike the original proposition [17], there is a guarantee that the heuristics of the geometric averaging with respect to the reference values always provides a feasible ranking result to the user. ${ }^{3}$

\subsection{Optimality condition}

One of the reasons for introducing the geometric mean method (3) is minimizing the multiplicative error $e_{i j}$ [9] defined as:

$$
m_{i j}=\frac{p_{i}}{p_{j}} e_{i j}
$$

In the case of the geometric averaging heuristics the multiplicative error equation takes the form:

$$
m_{i j}=\frac{\mu\left(c_{i}\right)}{\mu\left(c_{j}\right)} e_{i j}
$$

The multiplicative error is commonly accepted to be log normal distributed (in the same way the additive error would be assumed to be normally distributed). Let $e: \mathbb{R}_{+}^{n} \rightarrow \mathbb{R}$ be the sum of multiplicative errors (5). As is shown in the Theorem below the heuristics (9) is often optimal with respect to the value of the multiplicative error function $e$.

Theorem 2 The geometric averaging with respect to the reference values heuristics minimizes the sum of multiplicative errors $e\left(\mu\left(c_{1}\right), \ldots, \mu\left(c_{n}\right)\right)$ if

$$
\mu\left(c_{i}\right)<(n-1) \sum_{j=1, j \neq i}^{n} \mu\left(c_{j}\right)
$$

for $i=1, \ldots, n$.

Proof To determine the minimum of (5) let us forget for a moment that $\mu\left(c_{k+1}\right), \ldots, \mu\left(c_{n}\right)$ are constants (the reference values), and let us treat them as any other arguments of $e$. In order to determine the minimum of (5) the first derivative needs to be calculated. Thus,

$$
\begin{aligned}
\frac{\partial e}{\partial \mu\left(c_{i}\right)}= & \frac{1}{\mu\left(c_{i}\right)}\left(\sum_{r=1, r \neq i}^{n} 4(n-1) \ln \mu\left(c_{i}\right)-4 \sum_{j=1, j \neq i}^{n} \ln \mu\left(c_{j}\right)\right. \\
& \left.+2 \sum_{r=1, r \neq i}^{n} \ln \left(m_{r i}\right)-2 \sum_{j=1, j \neq i}^{n} \ln \left(m_{i j}\right)\right)
\end{aligned}
$$

3 The Monte Carlo tests recently carried out by the authors seem to indicate that the additive HRE approach fails for either non-reciprocal matrices (generalized PC matrices [15]), or the reciprocal PC matrices with the relatively high inconsistency, especially when $C_{K}$ is small. For instance $M=\left(\begin{array}{cccc}1 & 10 & 1 & 1 \\ 1 / 10 & 1 & 100 & 1 \\ 1 & 1 / 100 & 1 & 1,000 \\ 1 & 1 & 1 / 1,000 & 1\end{array}\right)$, where $C_{K}=\left\{c_{4}\right\}$ and $\mu\left(c_{4}\right)=1$, makes the additive method fails (a resulting solution has negative values). In this case the Saaty inconsistency index $\mathscr{S}(M)$ for $M$ is greater than 15 . Thus, in practice, such a matrix would have never been considered as a base to create a ranking. At the moment it is shown that the additive HRE provides a feasible solution when $M$ is consistent enough [18]. 
for $i=1, \ldots, n$. Due to the reciprocity of $M$, i.e. $m_{i j}=1 / m_{j i}$, the Eq. (21) can be written as:

$$
\frac{\partial e}{\partial \mu\left(c_{i}\right)}=-4\left(\frac{\sum_{j=1, j \neq i}^{n}\left(\ln \mu\left(c_{j}\right)+\ln \left(m_{i j}\right)\right)-(n-1) \ln \mu\left(c_{i}\right)}{\mu\left(c_{i}\right)}\right)
$$

The function $e$ reaches the minimum if $\partial e / \partial \mu\left(c_{i}\right)=0$. This leads to the postulate that

$$
\sum_{j=1, j \neq i}^{n}\left(\ln \mu\left(c_{j}\right)+\ln \left(m_{i j}\right)\right)-(n-1) \ln \mu\left(c_{i}\right)=0
$$

for $i=1, \ldots, n$. Thus,

$$
\ln \mu\left(c_{i}\right)=\frac{1}{n-1}\left(\sum_{j=1, j \neq i}^{n} \ln m_{i j} \mu\left(c_{j}\right)\right)
$$

which is directly equivalent to (9). In other words any solution to the equation system (10) is a good candidate to be a minimum of (5). It remains to settle the matrix $H$ of second derivative of $e$. When $H$ is positive definite then the solution of (10) actually minimizes the function $e$. As a result of further differentiation is determined that the diagonal elements of $H$ are

$$
\frac{\partial^{2} f}{\partial \mu\left(c_{i}\right) \partial \mu\left(c_{i}\right)}=\frac{4(n-1)}{\mu^{2}\left(c_{i}\right)}-\frac{1}{\mu\left(c_{i}\right)} \frac{\partial f}{\partial \mu\left(c_{i}\right)}
$$

where $i=1, \ldots, n$, and the other elements for which $i \neq j$ and $i, j=1, \ldots, n$ take the form:

$$
\frac{\partial^{2} f}{\partial \mu\left(c_{i}\right) \partial \mu\left(c_{j}\right)}=-\frac{4}{\mu\left(c_{i}\right) \mu\left(c_{j}\right)}
$$

Since the matrix $H$ is considered for $e$ in the point $\left(\mu\left(c_{1}\right), \ldots, \mu\left(c_{n}\right)\right)$ such that (9) holds, the first derivative of $e$ is 0 . Therefore, the Hessian matrix $H$ takes the form:

$$
H=\left[\begin{array}{cccc}
\frac{4(n-1)}{\mu^{2}\left(c_{1}\right)} & -\frac{4}{\mu\left(c_{1}\right) \mu\left(c_{2}\right)} & \cdots & -\frac{4}{\mu\left(c_{1}\right) \mu\left(c_{n}\right)} \\
\vdots & \frac{4(n-1)}{\mu^{2}\left(c_{2}\right)} & \vdots & \vdots \\
\vdots & \vdots & \ddots & \vdots \\
-\frac{4}{\mu\left(c_{n}\right) \mu\left(c_{1}\right)} & -\frac{4}{\mu\left(c_{n}\right) \mu\left(c_{2}\right)} & \cdots & \frac{4(n-1)}{\mu^{2}\left(c_{n}\right)}
\end{array}\right]
$$

According to [24, p. 29], if $H$ is strictly diagonally dominant by rows, symmetrical, and with positive diagonal entries, then it is also positive definite. To meet the first strict diagonal dominance criterion (others are satisfied) it is required that:

$$
\left|\frac{n-1}{\mu^{2}\left(c_{i}\right)}\right|>\sum_{j=1, j \neq i}^{n}\left|-\frac{1}{\mu\left(c_{i}\right) \mu\left(c_{j}\right)}\right|
$$

for $i=1, \ldots, n$. Thus,

$$
\mu^{2}\left(c_{i}\right)<(n-1) \mu\left(c_{i}\right) \sum_{j=1, j \neq i}^{n} \mu\left(c_{j}\right)
$$

Since every $\mu\left(c_{i}\right)>0$, it is easy to verify that the above equation is equivalent to the desired condition (20). 


\section{Numerical examples}

The HRE method can be useful in many situations in which, based on expert subjective opinions and actual data, new concepts, objects or entities need to be assessed. In order to show how the method might work in practice the following two numerical examples are presented. The first one, more abstract, discusses using the method to solve the non-linear equation system. The second, more complex, tries to put the method into an actual business context, where it can be successfully used.

In both examples the set of concepts consists of $C_{K}$-the reference (known) and $C_{U}$-the initially unknown elements. The numerical results ${ }^{4}$ were calculated using the PairwiseComparisons Mathematica $^{\mathrm{TM}}$ package. $^{5}$

\subsection{Example I (Scientific entities assessment)}

Let $c_{1}, \ldots, c_{5}$ represent research units, ${ }^{6}$ where two of them $\mathbf{c}_{2}, \mathbf{c}_{3} \in C_{K}$ are the reference units. Their values have been arbitrarily set by experts to $\mu\left(\mathbf{c}_{2}\right)=\mathbf{5}$ and $\mu\left(\mathbf{c}_{\mathbf{3}}\right)=\mathbf{7}$, whilst the other values $\mu\left(c_{1}\right), \mu\left(c_{4}\right)$ and $\mu\left(c_{5}\right)$ are considered unknowns. To determine the missing values the experts compare $c_{1}, \ldots, c_{5}$ in pairs and propose the values $m_{i j}$ defining the relative priority of $c_{i}$ with respect to $c_{j}$. An exception is the pair $\left(c_{2}, c_{3}\right)$ for which the ratios $m_{23}=$ $5 / 7$ and $m_{32}=7 / 5$ follow directly from the initially known values $\mu\left(c_{2}\right)$ and $\mu\left(c_{3}\right)$.

The expert analysis of the scientific achievements of the units $c_{1}, c_{4}$ and $c_{5}$ leads to the following $P C$ matrix ${ }^{7} M=\left[m_{i j}\right]$ :

$$
M=\left[\begin{array}{ccccc}
1 & \frac{3}{5} & \frac{4}{7} & 2 & \frac{16}{7} \\
\frac{5}{3} & 1 & \frac{5}{7} & \frac{5}{2} & \frac{10}{3} \\
\frac{7}{4} & \frac{7}{5} & 1 & \frac{7}{2} & 4 \\
\frac{1}{2} & \frac{2}{5} & \frac{2}{7} & 1 & \frac{4}{3} \\
\frac{7}{16} & \frac{3}{10} & \frac{1}{4} & \frac{3}{4} & 1
\end{array}\right]
$$

Hence, for example, according to the expert judgment the relative priority of $c_{1}$ with respect to $c_{2}$ is $m_{12}=3 / 5$. Since the matrix is reciprocal it holds that $m_{i j}=1 / m_{j i}$.

In accordance with the proposed hypothesis (9), to calculate the rank using HRE with the geometric averaging heuristics, the following system of non-linear equations (compare with 10) needs to be solved:

$$
\begin{aligned}
& \mu\left(c_{1}\right)=\left(m_{1,2} \mu\left(c_{2}\right) \cdot \ldots \ldots \ldots \ldots \ldots \ldots \cdot m_{1,5} \mu\left(c_{5}\right)\right)^{\frac{1}{4}} \\
& \mu\left(c_{4}\right)=\left(m_{4,1} \mu\left(c_{1}\right) \cdot \ldots \cdot m_{4,3} \mu\left(c_{1}\right) \cdot m_{4,5} \mu\left(c_{5}\right)\right)^{\frac{1}{4}} \\
& \mu\left(c_{5}\right)=\left(m_{5,1} \mu\left(c_{1}\right) \cdot \ldots \ldots \ldots \ldots \ldots \ldots \cdot m_{5,4} \mu\left(c_{4}\right)\right)^{\frac{1}{4}}
\end{aligned}
$$

thus, after raising both sides of the equations to the power,

$$
\begin{aligned}
& \mu^{4}\left(c_{1}\right)=m_{1,2} \mu\left(c_{2}\right) \cdot \ldots \ldots \ldots \ldots \ldots \ldots \cdot m_{1,5} \mu\left(c_{5}\right) \\
& \mu^{4}\left(c_{4}\right)=m_{4,1} \mu\left(c_{1}\right) \cdot \ldots \cdot m_{4,3} \mu\left(c_{1}\right) \cdot m_{4,5} \mu\left(c_{5}\right) \\
& \mu^{4}\left(c_{5}\right)=m_{5,1} \mu\left(c_{1}\right) \cdot \ldots \ldots \ldots \ldots \ldots \ldots \cdot m_{5,4} \mu\left(c_{4}\right)
\end{aligned}
$$

\footnotetext{
4 Available on line at www.kulakowski.org/pc.

5 Freely available at www.kulakowski.org/pcpackage/.

6 In fact, the official ranking of research units in Poland compares them in pairs [13].

7 The values a priori known are written in bold.
} 
Substituting the logarithm of both sides of the equations, we get the following system:

$$
\begin{aligned}
& 4 \lg \mu\left(c_{1}\right)=\lg \left(m_{1,2} \mu\left(c_{2}\right) \cdot \ldots \ldots \ldots \ldots \ldots \ldots \cdot m_{1,5} \mu\left(c_{5}\right)\right) \\
& 4 \lg \mu\left(c_{4}\right)=\lg \left(m_{4,1} \mu\left(c_{1}\right) \cdot \ldots \cdot m_{4,3} \mu\left(c_{1}\right) \cdot m_{4,5} \mu\left(c_{5}\right)\right) \\
& 4 \lg \mu\left(c_{5}\right)=\lg \left(m_{5,1} \mu\left(c_{1}\right) \cdot \ldots \ldots \ldots \ldots \ldots . m_{5,4} \mu\left(c_{4}\right)\right)
\end{aligned}
$$

which leads to the intermediate, linear logarithmic equation system:

$$
\begin{aligned}
& 4 \lg \mu\left(c_{1}\right)-\lg \mu\left(c_{4}\right)-\lg \mu\left(c_{5}\right)=b_{1} \\
& -\lg \mu\left(c_{1}\right)+4 \lg \mu\left(c_{4}\right)-\lg \mu\left(c_{5}\right)=b_{4} \\
& -\lg \mu\left(c_{1}\right)-\lg \mu\left(c_{4}\right)+4 \lg \mu\left(c_{5}\right)=b_{5}
\end{aligned}
$$

where

$$
\begin{aligned}
& b_{1} \stackrel{d f}{=} \lg \left(m_{1,2} \mu\left(c_{2}\right) m_{1,3} \mu\left(c_{3}\right) m_{1,4} m_{1,5}\right) \\
& b_{4} \stackrel{d f}{=} \lg \left(m_{4,1} m_{4,2} \mu\left(c_{2}\right) m_{4,3} \mu\left(c_{3}\right) m_{4,5}\right) \\
& b_{5} \stackrel{d f}{=} \lg \left(m_{5,1} m_{5,2} \mu\left(c_{2}\right) m_{5,3} \mu\left(c_{3}\right) m_{5,4}\right)
\end{aligned}
$$

Then, according to the procedure proposed in (Sect. 3.1), the linear equation system (14) where the unknown values $\hat{\mu}\left(c_{i}\right) \stackrel{d f}{=} \lg \left(\mu\left(c_{i}\right)\right)$ for $i=1,4,5$ takes the form:

$$
\left[\begin{array}{ccc}
n-1 & -1 & -1 \\
-1 & n-1 & -1 \\
-1 & -1 & n-1
\end{array}\right]\left[\begin{array}{l}
\widehat{\mu}\left(c_{1}\right) \\
\widehat{\mu}\left(c_{4}\right) \\
\widehat{\mu}\left(c_{5}\right)
\end{array}\right]=\left[\begin{array}{l}
b_{1} \\
b_{4} \\
b_{5}
\end{array}\right]
$$

hence, numerically:

$$
\left[\begin{array}{ccc}
4 & -1 & -1 \\
-1 & 4 & -1 \\
-1 & -1 & 4
\end{array}\right]\left[\begin{array}{l}
\widehat{\mu}\left(c_{1}\right) \\
\widehat{\mu}\left(c_{4}\right) \\
\widehat{\mu}\left(c_{5}\right)
\end{array}\right]=\left[\begin{array}{c}
1.739 \\
0.425 \\
-0.064
\end{array}\right]
$$

Solving the linear equation system provides us with $\widehat{\mu}\left(c_{1}\right)=0.557, \widehat{\mu}\left(c_{4}\right)=0.295$ and $\widehat{\mu}\left(c_{5}\right)=0.197$ which leads to the desired result $10^{\widehat{\mu}\left(c_{1}\right)}=3.613,10^{\widehat{\mu}\left(c_{4}\right)}=1.973$ and $10^{\widehat{\mu}\left(c_{5}\right)}=1.574$. The non-scaled weight vector $\mu$ supplemented by the known values $\mu\left(c_{2}\right)=5$ and $\mu\left(c_{3}\right)=7$ takes the form:

$$
\mu=[3.613, \mathbf{5}, \mathbf{7}, 1.973,1.574]^{T}
$$

and after rescaling:

$$
\mu_{n}=[0.189, \mathbf{0 . 2 6 1}, \mathbf{0 . 3 6 5}, 0.103,0.0822]^{T}
$$

Note that $\left|C_{U}\right|=3$ implies that the dimensions of matrix $\hat{A}$ are $3 \times 3$, moreover $\operatorname{det}(\hat{A}) \neq 0$ and $\mu\left(c_{i}\right)>0$ for $i=1,4,5$ (see Sect. 3.2). The inconsistency index is $\mathscr{S}(M)=0.003<$ 0.1 . Thus, according to Saaty criterion, the matrix is consistent enough to be a reliable input to the ranking procedure.

Forgetting for a moment that the values $\mu\left(c_{2}\right)$ and $\mu\left(c_{3}\right)$ are known, the ranking for $c_{1}, \ldots, c_{5}$ can be calculated on the basis of $\mathrm{M}$ only using the eigenvalue based and geometric mean methods $(2,4)$. The appropriate (scaled) weight vectors are: $\mu_{e v} \approx \mu_{g m}=[0.189$, $0.274,0.35,0.103,0.082]^{T}$. Solving the logarithmic least square optimization problem (5) using the Nelder-Mead method [21] with constraints $\mu\left(c_{2}\right) / \mu\left(c_{3}\right)=5 / 7$ and $\mu\left(c_{1}\right)>$ $0, \ldots, \mu\left(c_{5}\right)>0$, leads to the priorities $\mu_{n m}=[0.189,0.261,0.365,0.103,0.0822]^{T}$. The weight vector obtained using the HRE additive method [17] is $\mu_{H R E a}=[0.19,0.26,0.364$, $0.103,0.0824]^{T}$.

It is worth to note that all the rankings preserve the same order of preferences, i.e. $c_{3}$ gets the highest rank, next there are $c_{2}, c_{1}, c_{4}$ and $c_{5}$. They differ in the numeric details. As can be expected (Sect. 3.3) the vectors $\mu_{n}$ and $\mu_{n m}$ are almost identical. 
4.2 Example II (Choosing the best TV show)

A certain TV broadcaster wants to produce a new entertainment TV show in a European country. It considers purchasing the license for one of five different entertainment shows produced in the United States. So far in Europe three similar programs have been broadcasted. Through market research the approximate size of their European audience is known. They are 5,500,000, 4,500,000 and 4,950,000 persons for programs $c_{6}, c_{7}$ and $c_{8}$ respectively. The production costs of these programs are similar. In order to select possibly the most profitable TV show the station hires a few seasoned media experts. During the expert panel they prepare the following $P C$ matrix $M$, representing the relative attractiveness of all the considered programs.

$$
M=\left[\begin{array}{cccccccc}
1 & 0.8 & 0.968 & 0.7 & 0.5 & 0.682 & 0.908 & 0.667 \\
1.25 & 1 & 1.211 & 0.994 & 0.757 & 0.75 & 1.27 & 0.833 \\
1.033 & 0.826 & 1 & 0.821 & 0.551 & 0.704 & 0.826 & 0.625 \\
1.429 & 1.006 & 1.218 & 1 & 0.714 & 0.857 & 1.050 & 0.952 \\
2 & 1.321 & 1.817 & 1.401 & 1 & 1.2 & 1.467 & 1.333 \\
1.467 & 1.333 & 1.42 & 1.167 & 0.833 & 1 & \mathbf{1 . 2 2 2} & \mathbf{1 . 1 1 1} \\
1.101 & 0.8 & 1.211 & 0.952 & 0.682 & \mathbf{0 . 8 1 8} & 1 & \mathbf{0 . 9 0 9} \\
1.499 & 1.2 & 1.6 & 1.05 & 0.75 & \mathbf{0 . 9} & \mathbf{1 . 1} & 1
\end{array}\right]
$$

In the matrix $M$ every entry $m_{i j}$ corresponds to a ratio describing attractiveness of the $T V$ show $c_{i}$ with respect to the attractiveness of $T V$ show $c_{j}$. Since the values of attractiveness for $c_{6}, c_{7}$ and $c_{8}$ are known (they are approximated by the number of people watching the given TV show), thus the appropriate ratios $m_{i j}$ for $i, j=6,7,8$ are not the subject of expert judgment. Instead, they are calculated based on data from market research. For example:

$$
\mathbf{m}_{\mathbf{6}, 7}=\frac{\mu\left(c_{6}\right)}{\mu\left(c_{7}\right)}=\frac{5,100,000}{4,500,000}=\mathbf{1 . 2 2 2}
$$

or

$$
\mathbf{m}_{\mathbf{6}, \mathbf{8}}=\frac{\mu\left(c_{6}\right)}{\mu\left(c_{8}\right)}=\frac{5,100,000}{4,950,000}=\mathbf{1 . 1 1 1}
$$

The other entries of $M$ represent the subjective judgements of experts.

Like before, to find a solution with the help of HRE supported by the geometric averaging heuristics, the system of equations (10) must be solved. The desired values $\mu\left(c_{i}\right)$ for $i=1 \ldots, 5$ will be derived from the formula $\widehat{\mu}\left(c_{i}\right)=\log \mu\left(c_{i}\right)$. Because $\left|C_{U}\right|=5$, the dimensions of matrix $\widehat{A}$ are $5 \times 5$. The linear equation system need to be solved as follows:

$$
\left[\begin{array}{ccccc}
n-1 & -1 & -1 & -1 & -1 \\
-1 & n-1 & -1 & -1 & -1 \\
-1 & -1 & n-1 & -1 & -1 \\
-1 & -1 & -1 & n-1 & -1 \\
-1 & -1 & -1 & -1 & n-1
\end{array}\right]\left[\begin{array}{l}
\widehat{\mu}\left(c_{1}\right) \\
\widehat{\mu}\left(c_{2}\right) \\
\widehat{\mu}\left(c_{3}\right) \\
\widehat{\mu}\left(c_{4}\right) \\
\widehat{\mu}\left(c_{5}\right)
\end{array}\right]=\left[\begin{array}{l}
b_{1} \\
b_{2} \\
b_{3} \\
b_{4} \\
b_{5}
\end{array}\right]
$$

where

$$
\begin{aligned}
& b_{1} \stackrel{d f}{=} \lg \left(m_{1,2} m_{1,3} m_{1,4} m_{1,5} m_{1,6} \mu\left(c_{6}\right) m_{1,7} \mu\left(c_{7}\right) m_{1,8} \mu\left(c_{8}\right)\right) \\
& b_{2} \stackrel{d f}{=} \lg \left(m_{2,1} m_{2,3} m_{2,4} m_{2,5} m_{2,6} \mu\left(c_{6}\right) m_{2,7} \mu\left(c_{7}\right) m_{2,8} \mu\left(c_{8}\right)\right) \\
& b_{3} \stackrel{d f}{=} \lg \left(m_{3,1} m_{3,2} m_{3,4} m_{3,5} m_{3,6} \mu\left(c_{6}\right) m_{3,7} \mu\left(c_{7}\right) m_{3,8} \mu\left(c_{8}\right)\right) \\
& b_{4} \stackrel{d f}{=} \lg \left(m_{4,1} m_{4,2} m_{4,3} m_{4,5} m_{4,6} \mu\left(c_{6}\right) m_{4,7} \mu\left(c_{7}\right) m_{4,8} \mu\left(c_{8}\right)\right) \\
& b_{5} \stackrel{d f}{=} \lg \left(m_{5,1} m_{5,2} m_{5,3} m_{5,4} m_{5,6} \mu\left(c_{6}\right) m_{5,7} \mu\left(c_{7}\right) m_{5,8} \mu\left(c_{8}\right)\right)
\end{aligned}
$$


hence, (43) numerically:

$$
\left[\begin{array}{ccccc}
7 & -1 & -1 & -1 & -1 \\
-1 & 7 & -1 & -1 & -1 \\
-1 & -1 & 7 & -1 & -1 \\
-1 & -1 & -1 & 7 & -1 \\
-1 & -1 & -1 & -1 & 7
\end{array}\right]\left[\begin{array}{l}
\widehat{\mu}\left(c_{1}\right) \\
\widehat{\mu}\left(c_{2}\right) \\
\widehat{\mu}\left(c_{3}\right) \\
\widehat{\mu}\left(c_{4}\right) \\
\widehat{\mu}\left(c_{5}\right)
\end{array}\right]=\left[\begin{array}{l}
19.137 \\
20.037 \\
19.234 \\
20.117 \\
21.285
\end{array}\right]
$$

The intermediate result vector is:

$$
\widehat{\mu}=[6.551,6.664,6.563,6.674,6.82]^{T}
$$

Hence, following the rule $\mu\left(c_{i}\right)=\xi^{\widehat{\mu}\left(c_{i}\right)}$, where $\xi=10$ is the logarithm base, the final result vector is calculated.

$$
\mu=\left[\begin{array}{l}
3,556,858 \\
4,608,487 \\
3,657,533 \\
4,716,180 \\
6,601,181
\end{array}\right]
$$

The rescaled full priority vector is $\mu_{n}=[0.0934,0.121,0.096,0.124,0.173, \mathbf{0 . 1 4 4}$, $\mathbf{0 . 1 1 8}, \mathbf{0 . 1 3}]^{T}$. Thus, according to the expert judgments and the market research TV show number 5 (denoted as $c_{5}$ ) has the chance to gather nearly 6.5 million people in front of their TVs, whilst the second one in line can gather "only" 4.6 million people. Based on this estimate, the board of directors representing the broadcaster decides to recommend purchasing the license for the fifth presented TV show. Similarly as before $\mathscr{S}(M)=0.002$ is much smaller than 0.1 . Thus, the matrix $M$ seems to be consistent enough to recognize the result obtained as relevant.

The eigenvalue based and geometric mean ranks calculated for $M$ (solely) are $\mu_{e v} \approx$ $\mu_{g m}=[0.0933,0.121,0.0959,0.124,0.173,0.144,0.113,0.136]^{T}$. The HRE additive method for the pair $M$ and $C_{K}$ returns $\mu_{H R E a}=[0.093,0.122,0.096,0.124,0.173$, $\mathbf{0 . 1 4 4}, \mathbf{0 . 1 1 7}, \mathbf{0 . 1 2 9}]^{T}$. Solving the logarithmic least square optimization problem (5) using the Nelder-Mead method [21] with the additional constraints $\mu\left(c_{6}\right) / \mu\left(c_{7}\right)=1.222$, $\mu\left(c_{6}\right) / \mu\left(c_{7}\right)=1.111, \mu\left(c_{7}\right) / \mu\left(c_{8}\right)=0.909$, and $\mu\left(c_{1}\right)>0, \ldots, \mu\left(c_{8}\right)>0$, leads to the following priority vector: $\mu_{n m}=[0.0934,0.121,0.096,0.123,0.173,0.144,0.118$, $0.13]^{T}$. Similarly as before, all the vectors preserve the same rank order, which is $c_{5}, c_{6}, c_{8}$, $c_{4}, c_{2}, c_{7}$ and $c_{1}$. Similarly as before the vectors $\mu_{n}$ and $\mu_{n m}$ are almost identical.

\section{Summary}

The presented geometric HRE approach is another solution to the problem of rankings with the reference set. It proposes to use a geometric mean instead of the arithmetic one used in $[16,17]$. The advantage of this approach is the robustness of the procedure. As it was shown in (Sect. 3.2) the proposed solution works for an arbitrary set of input data producing an admissible vector of weights. The resulted ranking very often turns out to be optimal in the sense of the magnitude of multiplicative errors. According to the formulated and proven condition (Sect. 3.3), this happens when the differences between the resulting priorities are not too large. Therefore, the geometric HRE approach is also a method of solving the logarithmic least square constrained optimization problem.

The HRE approach may be useful in many different situations, including ranking creation, valuation of goods and services, risk assessment and others. Due to the lack of restrictions 
on the input $P C$ matrix (there is a guarantee that a method with the geometric mean always produces an admissible result), the scope of the applicability of the HRE method increases. Thus, the presented method covers cases (e.g. rankings with non-reciprocal PC matrices) which can not always be dealt with using the arithmetic mean heuristics. The HRE method may also be useful for the AHP users. In this context, for the non-empty reference set $C_{K}$, it may serve as an alternative for the standard priority deriving methods such as eigenvalue based method [25] or geometric mean method [6].

Despite these encouraging results, much remains to be done. In particular, the role of the inconsistency in the input matrix $M$ should be more deeply investigated. Also the existence of solution for the "additive" HRE approach needs to be better researched. Of course, the more examples are studied the better. Thus, further development of the method will be particularly focused on the study and analysis of examples of its use.

Acknowledgments Authors are grateful to the Academic Computer Center Cyfronet AGH for providing the Wolfram Mathematica $^{\mathrm{TM}}$ software. Special thanks due to the Dan Swain for his editorial help.

Open Access This article is distributed under the terms of the Creative Commons Attribution License which permits any use, distribution, and reproduction in any medium, provided the original author(s) and the source are credited.

\section{References}

1. Bozóki, S., Fülöp, J., Rónyai, L.: On optimal completion of incomplete pairwise comparison matrices. Math. Comput. Model. 52(1-2), 318-333 (2010). doi:10.1016/j.mcm.2010.02.047

2. Bozóki, S., Rapcsak, T.: On Saaty's and Koczkodaj's inconsistencies of pairwise comparison matrices. J. Glob. Optim. 42(2), 157-175 (2008)

3. Brunelli, M., Canal, L., Fedrizzi, M.: Inconsistency indices for pairwise comparison matrices: a numerical study. Ann. Oper. Res. 211, 493-509 (2013). doi:10.1007/s10479-013-1329-0

4. Brunelli, M., Fedrizzi, M.: Axiomatic properties of inconsistency indices. J. Oper. Res. Soc. (2013). doi:10.1057/jors.2013.135

5. Colomer, J.M.: Ramon Llull: from 'Ars electionis' to social choice theory. Soc. Choice Welf. 40(2), 317-328 (2011)

6. Crawford, G.B.: The geometric mean procedure for estimating the scale of a judgement matrix. Math. Model. 9(3-5), 327-334 (1987). doi:10.1016/0270-0255(87)90489-1

7. Faliszewski, P., Hemaspaandra, E., Hemaspaandra, L.A., Rothe, J.: Llull and Copeland voting computationally resist bribery and constructive control. J. Artif. Intell. Res. (JAIR) 35, 275-341 (2009)

8. Fülöp, J., Koczkodaj, W.W., Szarek, S.J.: A different perspective on a scale for pairwise comparisons. Trans. Comput. Collect. Intell. 1, 71-84 (2010)

9. Ishizaka, A., Labib, A.: Review of the main developments in the analytic hierarchy process. Expert Syst. Appl. 38(11), 14,336-14,345 (2011)

10. Ishizaka, A., Lusti, M.: How to derive priorities in AHP: a comparative study. Cent. Eur. J. Oper. Res. 14(4), 387-400 (2006). http://ideas.repec.org/a/spr/cejnor/v14y2006i4p387-400.html

11. Janicki, R., Zhai, Y.: On a pairwise comparison-based consistent non-numerical ranking. Log. J. IGPL 20(4), 667-676 (2012)

12. Kacprzyk, J., Zadrozny, S., Fedrizzi, M., Nurmi, H.: On group decision making, consensus reaching, voting and voting paradoxes under fuzzy preferences and a fuzzy majority: a survey and some perspectives. In: Studies in Fuzziness and Soft Computing, Studies in Fuzziness and Soft Computing, vol. 220, pp. 263-295. Springer, Berlin (2008)

13. Koczkodaj, W., Kułakowski, K., Ligęza, A.: On the quality evaluation of scientific entities in Poland supported by consistency-driven pairwise comparisons method. Scientometrics 99(3), 911-926 (2014). doi:10.1007/s11192-014-1258-y

14. Koczkodaj, W.W.: A new definition of consistency of pairwise comparisons. Math. Comput. Model. 18(7), 79-84 (1993). doi:10.1016/0895-7177(93)90059-8

15. Koczkodaj, W.W., Orłowski, M.: Computing a consistent approximation to a generalized pairwise comparisons matrix. Comput. Math. Appl. 37(3), 79-85 (1999) 
16. Kułakowski, K.: A heuristic rating estimation algorithm for the pairwise comparisons method. Cent. Eur. J. Oper. Res. 1-17 (2013). doi:10.1007/s10100-013-0311-x

17. Kułakowski, K.: Heuristic rating estimation approach to the pairwise comparisons nethod. Fundam. Inform. (to be appeared) 133, 367-386 (2014). doi:10.3233/FI-2014-1081

18. Kułakowski, K.: Notes on the existence of solutions in the pairwise comparisons method using the heuristic rating estimation approach. CoRR abs/1402.4064 (2014)

19. Lotfi, F.H., Fallahnejad, R., Navidi, N.: Ranking efficient units in DEA by using TOPSIS method. Appl. Math. Sci. (2011)

20. Mikhailov, L.: Deriving priorities from fuzzy pairwise comparison judgements. Fuzzy Sets Syst. 134(3), 365-385 (2003)

21. Nelder, J.A., Mead, R.: A simplex-method for function minimization. Comput. J. 7(4), 308-313 (1965)

22. Peterson, G.L., Brown, T.C.: Economic valuation by the method of paired comparison, with emphasis on evaluation of the transitivity axiom. Land Econ. 240-261 (1998)

23. Plemmons, R.J.: M-matrix characterizations. I-nonsingular M-matrices. Linear Algebra Appl. 18(2), 175-188 (1976)

24. Quarteroni, A., Sacco, R., Saleri, F.: Numerical Mathematics. Springer, Berlin (2000)

25. Saaty, T.L.: A scaling method for priorities in hierarchical structures. J. Math. Psychol. 15(3), 234-281 (1977). doi:10.1016/0022-2496(77)90033-5

26. Saaty, T.L.: The analytic hierarchy and analytic network processes for the measurement of intangible criteria and for decision-making. In: Multiple Criteria Decision Analysis: State of the Art Surveys, International Series in Operations Research and Management Science, vol. 78, pp. 345-405. Springer, New York (2005). doi:10.1007/0-387-23081-5_9

27. Saaty, T.L.: On the measurement of intangibles. A principal eigenvector approach to relative measurement derived from paired comparisons. Not. Am. Math. Soc. 60(02), 192 (2013)

28. Thurstone, L.L.: A law of comparative judgment, reprint of an original work published in 1927. Psychol. Rev. 101, 266-270 (1994) 